\title{
Fronteras europeas: De lo histórico a lo cotidiano
}

\author{
Borders in Europe: From history to everyday life
}

Recibido el 5 de febrero de 2016. Aceptado el 17 de noviembre de 2016.

Autor para correspondencia: Narcís Bassols, correo electrónico: narcis.bassols@gmail.com

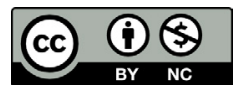

Todos los contenidos de Estudios Fronterizos se publican bajo la licencia Creative Commons Atribución no comercial 2.5 México, y pueden ser usados gratuitamente para fines no comerciales, dando el crédito a los autores y a la revista Estudios Fronterizos.

\author{
Narcís Bassols $\mathrm{a}^{*}(\mathbb{D}$ (http://orcid.org/0000-0001-5769-5323) \\ José Rafael Correa Fernández ${ }^{\mathfrak{B}}$ (http://orcid.org/0000-0002-9117-2064)
}

${ }^{a}$ Universidad Autónoma del Caribe. Programa de Administración de Empresas, Colombia, correo electrónico: narcis.bassols@gmail.com

${ }^{\mathrm{b}}$ Corporación Unificada Nacional de Educación Superior, Santa Marta, Colombia, correo electrónico: jose.rafael.correa@gmail.com

\section{Resumen}

El presente artículo reflexiona sobre fronteras europeas y sus dinámicas cotidianas desde una óptica transfronteriza. Se parte de algunos hechos recientes que cuestionan la actual política de fronteras de la Unión Europea para hacer a continuación un repaso de la literatura sobre fronteras y territorios fronterizos. Posteriormente, se discuten los aspectos cotidianos que las prácticas transfronterizas tienen para los habitantes de los espacios fronterizos. En algunas ocasiones, y para completar el cuadro, se hace referencia a fronteras americanas. Si bien el tema de la seguridad es relevante, nos enfocamos en las dimensiones económicas y sociales de la cooperación. Tres aspectos devienen esenciales para los habitantes de frontera, que pretenden usar las ventajas del territorio en su globalidad: trabajo, residencia y compras. Como conclusión, se puede afirmar que ha habido una tendencia hacia un suavizamiento de las fronteras y una mayor integración de las zonas fronterizas de la Unión Europea.

Palabras claves: frontera, espacio transfronterizo, integración fronteriza, Unión Europea, espacio Schengen.

\section{Abstract}

This article reflects on European borders and their everyday dynamics from a cross-border perspective. The article begins with a discussion of recent events that question current border policies in the European Union, reviews the literature on borders and border territories, and finally moves on to a discussion of the everyday effects of cross-border practices on the inhabitants of border spaces. Occasional reference is made to borders in the Americas to complete the picture of this topic. Although the issue of security is indeed relevant, we focus on the economic and social dimensions of cooperation. Three aspects are essential for border residents who attempt to use the advantages of the territories for their global nature: work, shopping,

CÓMO CITAR: Bassols, N. y Correa, J. R. (2017). Fronteras europeas: De lo histórico a lo cotidiano [Borders in Europe: From history to everyday life]. Estudios Fronterizos, 18(35), 131-149, doi: 10.21670/ref.2017.35.a7 
and place of residence. In conclusion, there has been a tendency toward the softening of borders and greater integration of border zones in the European Union.

Keywords: border, cross-border space, border integration, European Union, Schengen Area.

\section{Introducción}

El presente artículo es una revisión literaria conducente a una reflexión sobre las prácticas transfronterizas en Europa. Vista la disparidad de sentimientos que las fronteras suscitan, y las crisis fronterizas que casi a diario se reportan en Europa ${ }^{1}$ se impone una reflexión a fondo y sosegada a la que el presente artículo pretende contribuir, aportando sobre todo la larga, pionera y "mayormente" exitosa experiencia de la Unión Europea (UE) en el ámbito de la apertura de fronteras y la cooperación transfronteriza.

Evidentemente, la actual tendencia hacia lo que parece ser un repliegue en sí mismos de los Estados que configuran la UE tiene diversas lecturas, ya sea en clave política, social o histórica. En la presente contribución se va a razonar desde la espacialidad, la sociopolítica y la psicología para aportar otro tipo de soluciones que no sean el mencionado repliegue. Dichas soluciones creemos que están en la cooperación entre los países de la uE, y, más específicamente, entre sus áreas de frontera. Es por ello que preconizamos los proyectos transfronterizos como punta de lanza de una colaboración más amplia y nos parece interesante estudiarlos a fondo.

En el presente trabajo, en forma de artículo de reflexión, se presenta una doble visión de las fronteras europeas: por un lado la visión histórico-funcional, con una revisión de la literatura sobre fronteras y diversos aspectos transfronterizos, con foco especial en Europa; por otro lado, la segunda sección presenta algunos temas de actualidad en torno a modalidades de integración de las zonas transfronterizas del continente europeo. El artículo inicia constatando el fuerte cuestionamiento de la actual política europea de fronteras para posteriormente, tras una revisión de literatura y conceptos, dar algunas propuestas actuales del fenómeno transfronterizo tal como están siendo estudiadas por la academia europea. Este artículo es en parte fruto de las investigaciones y trabajos realizados en el marco del proyecto europeo transfronterizo Pyrémed-Pirimed ${ }^{2}$, cuya acción estuvo centrada en las regiones de frontera franco-españolas de la vertiente mediterránea, que duró hasta 2010 y ha servido para establecer algunas buenas prácticas transfronterizas que también se comunican.

A pesar de querer dar una visión amplia de las cuestiones mencionadas, debemos poner límites y alcances al presente artículo. Uno de ellos es que los casos que se ilustran se limitan prácticamente a Europa, con algunas escasas menciones a América, pero es un artículo básicamente sobre problemas europeos desde una óptica europea. Del mismo modo, se ofrecen experiencias europeas de cooperación transfronteriza y no de otros territorios del mundo. Por otro lado, nos interesan fundamentalmente los aspectos más económicos y sociales de la cooperación que los aspectos de seguridad: nuestro trabajo en el proyecto Pyrémed-Pirimed se centró en favorecer intercambios económico-sociales

\footnotetext{
1 En el momento de revisar este artículo (noviembre de 2016), se está desmantelando en la ciudad de Calais, en el norte de Francia, un gran campamento de refugiados que pretenden llegar al Reino Unido.

2 PYREnees MEDiterranee-PIRIneos MEDiterraneo, denominación bilingüe francés-español del área geográfica que abarcó el proyecto.
} 
y no en la seguridad. Con ello, no pretendemos dejar este aspecto en un segundo lugar, simplemente nuestra experticia está en otro ámbito, lo cual hacemos extensivo también a la cooperación internacional más allá de las fronteras de la UE. Finalmente, nos interesan más las prácticas cotidianas y lo que las determina, que los grandes proyectos o leyes que se ponen en marcha para favorecer la cooperación. Damos pues unas experiencias con algunas buenas prácticas para favorecer intercambios, pero esperamos que el lector de otras partes del mundo sepa dilucidar la conveniencia de transferir estas experiencias a su país.

\section{Algunos cuestionamientos actuales de la política de fronteras europeas del espacio Schengen}

Cualquier ciudadano europeo del espacio Schengen - en especial los que viven en los territorios de frontera- puede constatar la practicidad que significa a nivel cotidiano la aplicación de los principios de libre circulación de bienes, personas y servicios que propugna dicho espacio. Por otro lado, la misma ciudadanía constata sorprendida la fragilidad de dicho entramado político. Ello sucede actualmente con la crisis de los refugiados del Medio Oriente que llegan a territorio europeo, una crisis que lleva ya muchos meses en los medios informativos europeos y ha abierto profundas brechas y desacuerdos entre países del espacio Schengen con incluso algunos casos de reintroducción de controles fronterizos. No pocos comentaristas políticos sostienen que el proceso de salida del Reino Unido de la ue (brexit) puede entenderse en parte desde una óptica de manejo nacional de fronteras y flujos migratorios.

La cuestión ha llegado tan lejos que incluso France Stratégie, el laboratorio de ideas del gobierno francés, ha emitido un reporte calculando los perjuicios económicos de la reintroducción de los controles fronterizos permanentes. Según este informe, las pérdidas para Francia en el largo plazo serían de unos 10000 millones de euros, y de unos 100000 millones para toda la zona Schengen. De acuerdo con el informe, de una hipotética reintroducción de controles fronterizos, los más perjudicados serían los turistas de corta estadía, los estudiantes en intercambio, los trabajadores transfronterizos y los transportes de mercancías entre países (Aussilloux, 2016, p. 7).

A principios del año de 2014, otro hecho sorprendió la opinión pública europea: Suiza a través de referéndum decidió restringir la entrada de inmigrantes (europeos o no), contradiciendo de este modo el principio de libertad de movimiento de personas que consagra el espacio Schengen. Desde entonces, el país busca el modo de resolver esta contradicción a través de vericuetos legales, ya que de hecho el gobierno de ese país debe renegociar a la baja los tratados con UE respecto a la libre circulación de personas y la inmigración. Muchos medios de comunicación europeos dieron amplia cobertura a tal hecho porque significó de facto un retroceso en uno de los derechos de mayor impacto que se han establecido en la región en las últimas décadas. ${ }^{3}$ Un periódico español tituló "Suiza da un portazo a la Unión Europea" (Abellán, 2014); y un periódico suizo titulaba por su parte "Retroceso a los años 90" (Kohli, 2014). La decisión suiza abrió interrogantes sobre la idoneidad de tal medida ya que la economía de este país precisa mano de obra extranjera — cualificada o no- para seguir creciendo. Asimismo, esta situación llevó a

${ }^{3}$ Suiza no es miembro de la UE pero ha suscrito con esta acuerdos de cooperación y libre circulación, formando parte del espacio Schengen. 
reflexionar a otros gobiernos europeos sobre la libre circulación de personas (Bassets, 2014) en un momento en el que el populismo está en alza en Europa debido a la crisis económica. Ello para desespero de la Comisión Europea, que vio cómo la libre circulación de personas en territorio europeo, —uno de los pilares fundamentales sobre los que se ha construido la UE-, empieza a mostrar inestabilidad desde entonces (Figura 1).

\section{Figura 1: Resultados del voto popular suizo de febrero, 2014}

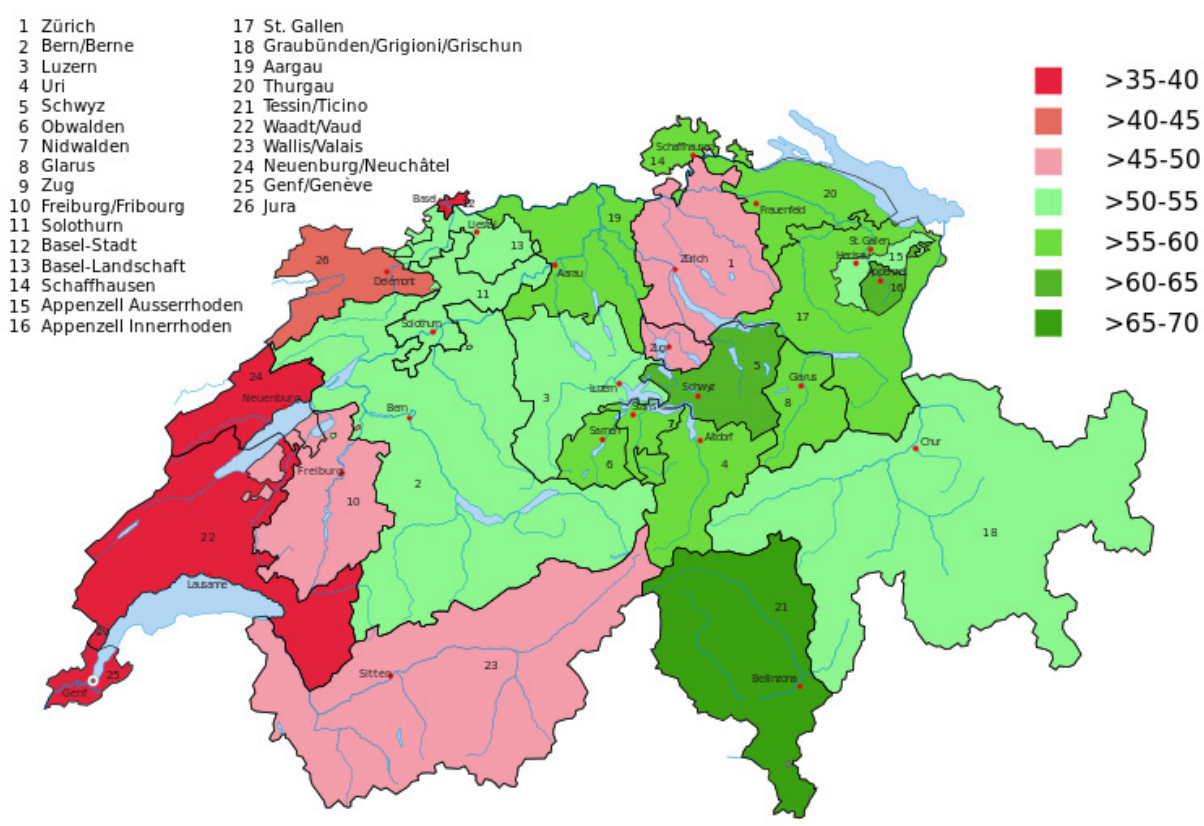

Nota: En tonos verdes, los cantones partidarios de limitar la inmigración. En tonos rojos, los contrarios. Se ve claramente la división entre los cantones de habla francesa del oeste del país así como de las grandes ciudades de Zurich y Basilea, versus el resto del país, mucho menos necesitado de mano de obra extranjera para sus economías locales.

Fuente: Wikipedia (2014).

El voto suizo es difícilmente comprensible ya que se puede afirmar que Suiza es un claro ejemplo de conurbaciones y regiones transfronterizas. Las ciudades de Ginebra y Basilea, segunda y tercera del país por población, con sus aeropuertos transnacionales incluidos, son las capitales del Grand Genève (que comprende tanto cantones suizos como departamentos franceses) y del Eurodistrito Trinacional de Basilea que engloba territorio de Suiza, Francia y Alemania, puesto que la ciudad de Basilea se halla en el triángulo fronterizo que forman estos tres países. El desarrollo de estas dos áreas metropolitanas se hace de modo transfronterizo; es decir, las autoridades locales de todos los territorios tienen voz sobre la planificación territorial. Ello ha hecho surgir modos de gobernanza muy novedosos. Se trata además de áreas transfronterizas con "larga tradición" ya que se institucionalizaron en los años sesenta del siglo pasado y por lo tanto hablamos de cinco décadas de cooperación transfronteriza (Figuras 2 y 3 ). ${ }^{4}$

${ }^{4}$ Por supuesto, no son las dos metrópolis suizas mencionadas las únicas áreas urbanas transfronterizas de Europa: el Proyecto Europeo URBACT ha identificado más de 60 áreas metropolitanas transfronterizas en Europa 
Figura 2: La ciudad de Ginebra (Genève) y su área metropolitana

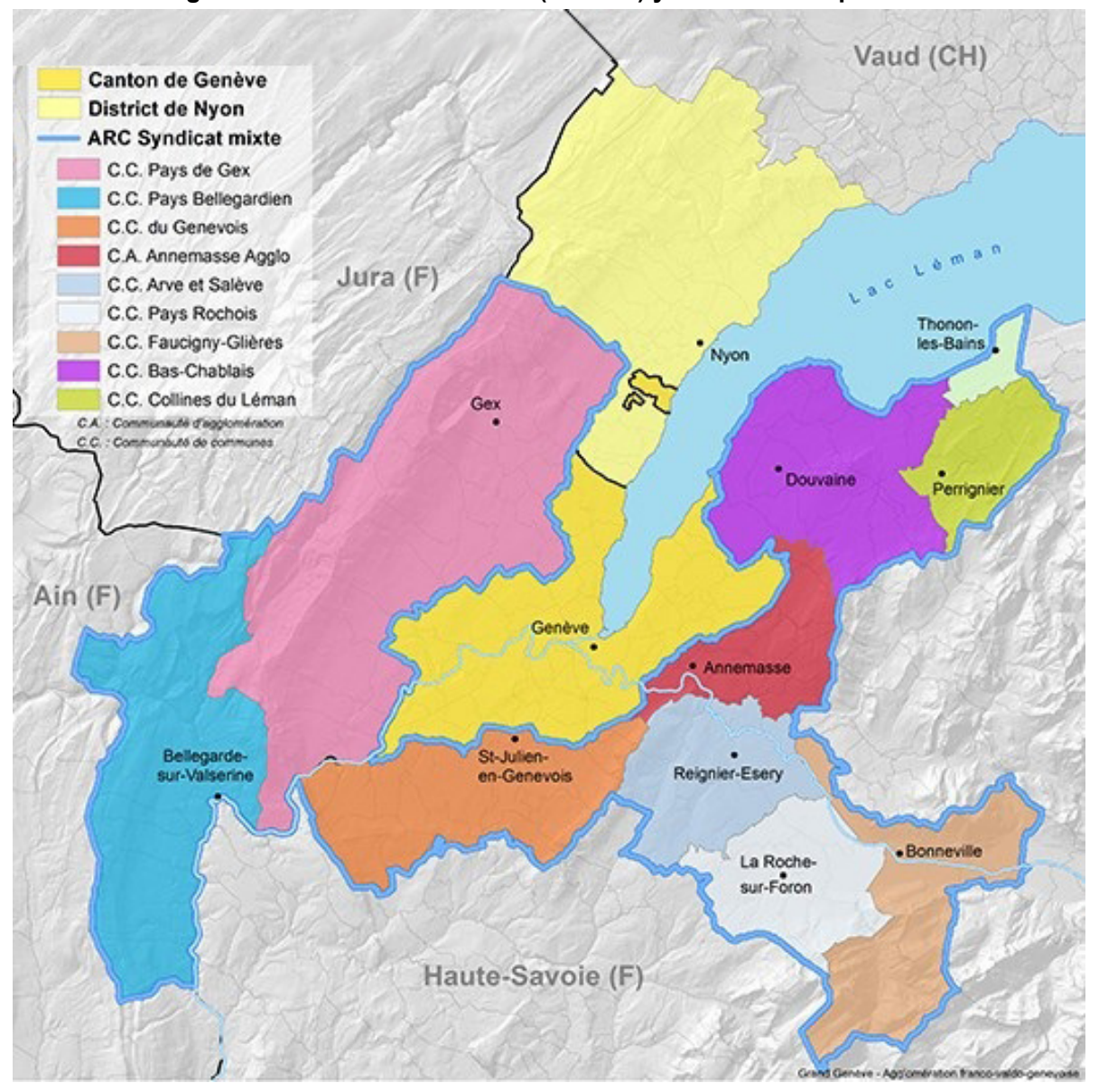

Nota: En tonos amarillo y beige, los territorios suizos que forman parte de esta área. El resto de territorios dibujados son las asociaciones de municipios franceses que forman parte de dicha área metropolitana.

Fuente: Cortesía del Grand Genève.

Figura 3: Vista aérea de Basilea y su área metropolitana

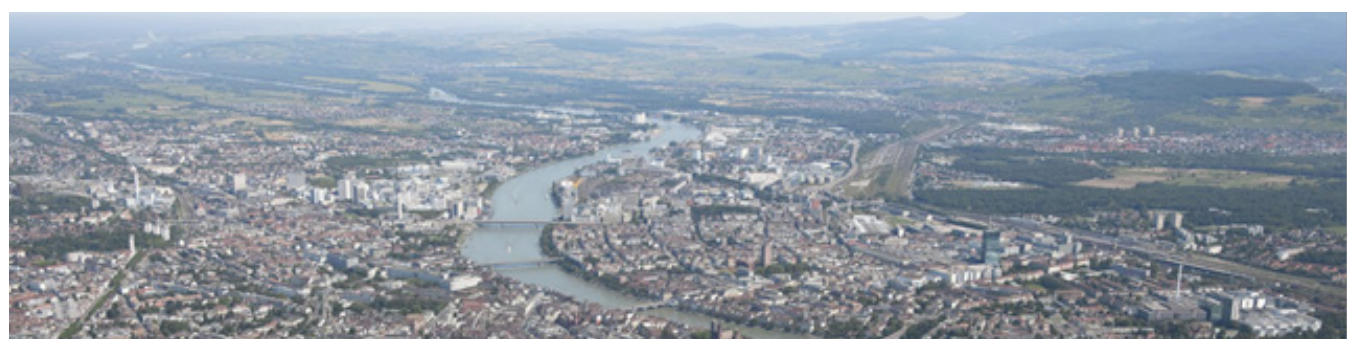

Nota: Área diseminada por tres países: Suiza, Francia y Alemania, en el centro se aprecia el río Rin, frontera natural y a la vez eje vertebrador.

Foto por: Fuerza Aérea Suiza en 2013.

en las que viven unos 25 millones de personas. Estas cifras dan una idea de la importancia y el reto que, dentro de la UE, significa articular correctamente todas estas áreas metropolitanas para hacerlas atractivas y competitivas, a pesar de su fragmentación por fronteras nacionales (URBACT, 2010). 
Si cambiamos el nivel metropolitano por el regional en Suiza, un buen ejemplo de cooperación interregional en dicho país es la zona natural y económica del Lago de Constanza, en el nordeste suizo, que se ha constituido desde hace años en un importante polo de desarrollo y de intercambio en el que participan, además de Suiza, los otros tres países ribereños del lago, esto es: Alemania, Austria y el principado de Liechtenstein.

Suiza es uno de los países de Europa con más movilidad de trabajadores transfronterizos. ${ }^{5}$ Y ello a pesar de su no-pertenencia a la UE, aunque sí forma parte de la Zona de Libre Comercio Europea (EFTA por sus siglas en inglés). Al país entran cada día a trabajar 143000 franceses, 62000 italianos, 56000 alemanes y 8100 austriacos, lo cual da la cifra de 270 000 personas. Por otra parte, la población suiza es de ocho millones de personas, con $23 \%$ de extranjeros, un porcentaje que en Europa solo supera Luxemburgo. En 2013 recibió unos 80000 inmigrantes procedentes mayormente de los países del sur de Europa, los cuales se hallan en crisis económica (Figura 4) (Poch, 2014).

Figura 4: Flujos de trabajadores transfronterizos en la región trinacional franco-germano-suiza

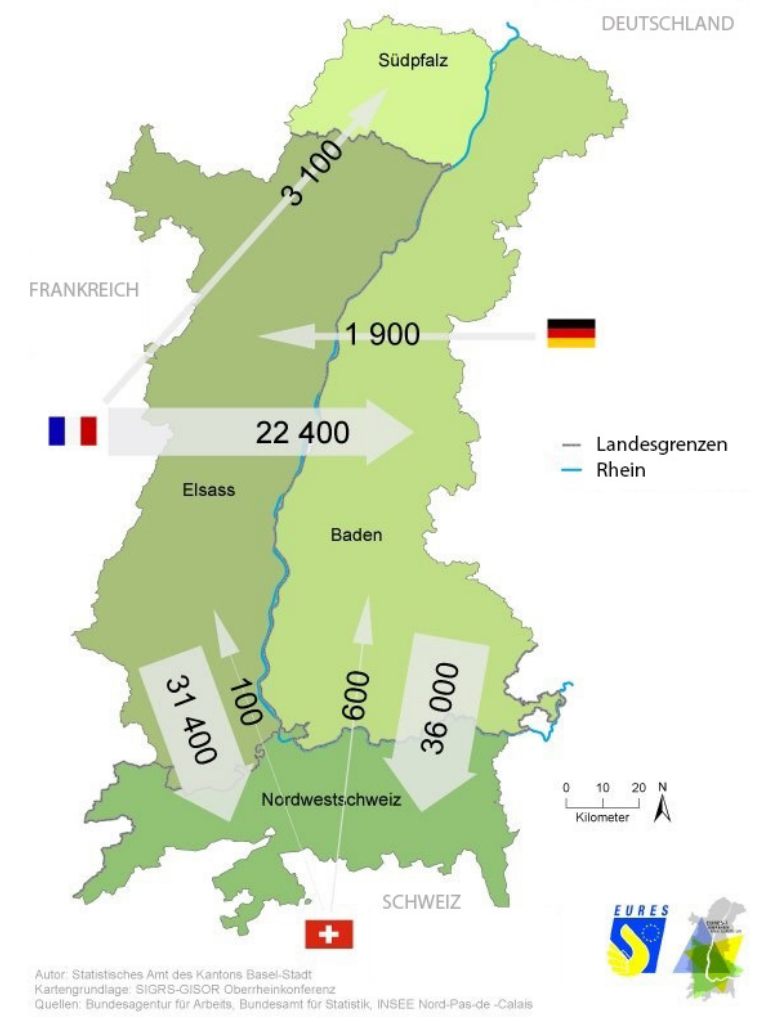

Nota: Se aprecia que Francia es el mayor emisor de trabajadores transfronterizos mientras que Suiza emite un número de trabajadores negligible y en cambio es gran receptora de un flujo de 95500 trabajadores transfronterizos en total.

Fuente: Cortesía de la Autoridad Estadística del Cantón de Basilea-Ciudad, Suiza (Regio Basiliensis, 2015, p.15).

\footnotetext{
${ }^{5}$ Técnicamente, la Unión Europea llama "trabajador transfronterizo" a aquel nacional que trabaja en un país vecino y que regresa a su domicilio en su país de origen por lo menos una vez a la semana. Esto último lo distingue del trabajador emigrante temporal. Pero de hecho, muchos trabajadores transfronterizos en Europa son commuters diarios, es decir, cruzan la frontera a diario para ir a sus puestos de trabajo desde su domicilio, que se halla en el país colindante.
} 
Con la importancia económica y social que la cooperación transfronteriza tiene para el país helvético, pocos entendieron pues el resultado del mencionado referéndum. Dicho resultado, analizado detenidamente, tiene claves de lectura tanto externas hacia Europa o el mundo (el miedo de un pequeño país a verse diluido en el magma uniformizador europeo) como internas (el ajustadísimo resultado significa una división interna en el país entre los cantones de habla francesa y las grandes urbes contra el resto del territorio, por los diferentes resultados locales de las votaciones). El mandato popular, además, rompió con dos décadas de integración de Suiza a la ue, que se ha dado gracias la sucesiva adhesión del país helvético a los diferentes tratados europeos de libre comercio y seguridad.

\section{De las fronteras y los territorios fronterizos al espacio transfronterizo: contextualización y enfoques}

Una aproximación inicial nos lleva a definir las fronteras como parte del entramado territorial y político de los pueblos. Sin embargo, en su forma actual, son fruto de cómo las entendió el Estado nación desde el nacimiento de este. Su utilidad ha sido básicamente funcionar como línea de demarcación de la soberanía de un país, de defensa y de separación hacia el exterior (cultural, comercial o militar). Por ello han sido siempre objeto de vigilancia más o menos estricta. Estas funciones han hecho de la frontera un instrumento más de la construcción de la identidad nacional (Blake, citado en Dubois y Rérat, 2012). En el plano económico, baste recordar el gran dilema histórico entre proteccionismo y librecambismo, un debate siempre "suavizado" sobre el terreno por el contrabando. Las fronteras son objeto del derecho internacional o la geografía política, y como tales han sido estudiadas largamente, véase Fawcett (1918) para un tratado de principios del pasado siglo siguiendo la aproximación geopolítica, Sahlins (1990) para una descripción histórico-evolutiva de las fronteras francesas o Gibler (2012) para una visión de la frontera como punto conflictivo.

Tradicionalmente, los territorios de frontera han sido tierras de paso, de múltiples identidades y de procesos socioeconómicos que no necesariamente han coincidido con los del resto del país. No pocas veces, dichos territorios, alejados del centro, presentan diferencias sociales notables respecto dicho centro y de la cultura mayoritaria de un país. Esto último es perfectamente comprobable por ejemplo en España o Francia, con sus periferias lingüísticamente diferenciadas (catalán, gallego y euskera en España; euskera, catalán, alemán, occitano o bretón en el caso de Francia). Según algunos autores, los territorios de frontera suelen presentar identidades propias más o menos fuertes, como se constata en la frontera entre Estados Unidos y México (Romo, 1987). Márquez y Romo (2008, p.1) citan a una residente de la frontera estadounidense-mexicana diciendo que, "There are three worlds here. You have the Mexican, the American, and the one here at the border, because here you have a combination of both cultures and it's different from the Mexican and the American". Incluso en casos de una contigüidad cultural y lingüística emergen identidades transfronterizas propias. Ello sucede en la frontera colombo-venezolana (Bustamante y Chacón, 2013) o en la comarca hispano-francesa de la Cerdanya en los Pirineos (Moncusí, 2008, p. 63 y ss.). Sin embargo, no es nuestra experiencia en el proyecto Pirimed que surjan dichas identidades, creemos que se trata de algo mucho más básico, esto es, de las habilidades que desarrollan los habitantes de regiones fronterizas para saber moverse del otro lado de la frontera, unas habilidades que tienen más que ver con la ampliación del propio ámbito geoespacial que con la creación de identidades mixtas como algunos han querido ver. 
Otro aspecto relevante de las regiones de frontera es el de la seguridad (un aspecto militar-estratégico crucial) y sus implicaciones económicas: con el concepto de frontera estratégica desarrollado por varios autores, los territorios de frontera adquieren una mayor relevancia militar que otras zonas del propio país. De ahí que, durante siglos, hayan sido tierras con una fuerte presencia militar (baste recordar la serie de fortalezas diseñadas por el arquitecto militar francés Vauban en las fronteras de la Francia de finales del siglo XVII $)^{6}$ y por lo tanto han sido consideradas como zonas menos seguras. Siguiendo esta lógica, los territorios de frontera se han visto no pocas veces en desventaja económica respecto al resto del país; en una visión de confrontación, ningún país querrá ubicar cerca de la frontera industrias clave, nadie planificará grandes urbes en estas regiones y las inversiones o las infraestructuras serán menores. Petrakos y Topaloglou (2006, p. 153) representan esta visión economicista: "Borders and border regions have been traditionally characterized as low opportunity areas hosting less advanced local economies". Esto explica, en parte, la dicotomía económica entre el centro y la periferia, muy extendida todavía en el mundo actual (Bertinat, Calvette, Ignatczuk, Sauval y Villamil, 2012). En países como Colombia, esta dicotomía entre el centro y las periferias se ha querido ilustrar con la figura del "triángulo de oro", que es el formado por las ciudades de Bogotá, Medellín y Cali. Una versión ampliada de este triángulo es el "polígono de oro", que incluye entonces las ciudades de Bucaramanga y Barranquilla.

La bipolaridad centro-periferia pues, en su triple vertiente cultural, económica y militar, ha marcado tradicionalmente los territorios transfronterizos, poniéndolos en clara desventaja respecto a otros territorios del mismo país. Dicho efecto, junto con otros que actúan en las zonas fronterizas, se han agrupado en lo que en la jerga burocrática de la UE se denomina de modo algo impreciso, como el efecto frontera y que condensa la desventaja de estas regiones respecto al resto de un país.

Las fronteras han evolucionado a lo largo de la historia (Bottino, 2009; Montero, 1997; Williams, 2006). Pero no es hasta mediados del siglo pasado, con el advenimiento de la globalización, y hasta la actualidad, en donde vemos que el espacio fronterizo gana importancia respecto a la frontera (véase el revelador título de Harguindéguy, 2007, $\mathrm{La}$ frontière en Europe: un territoire?), y esta por tanto pasa a ser dinámica y no estática: interesan más los intercambios y los flujos que tienen lugar por encima de las fronteras (Schuler, 2006; Koff, 2008). Entonces, las fronteras devienen, según algunos autores, "suaves" o "porosas" y se vuelven nexos de unión y no de separación, como reza el título de Comte y Levrant (2006): Aux coutures de l'Europe. La discusión fronteriza es hoy en día (por lo menos en Europa) no una cuestión de diferendos territoriales sino una discusión de cómo se planifica y organiza el espacio fronterizo de modo coherente, más allá de las divisorias entre países (Hinfray, 2010, p. 45 y ss.). Y según Bassols (2010, p. 47), las zonas fronterizas han evolucionado: "de zones de confrontació a laboratoris privilegiats de la construcció d'una Europa quotidiana”.

En este contexto de apertura europea de fronteras y de prevalencia de espacios transfronterizos, los intercambios económicos aumentan, seguidos de los intercambios sociales (en un orden de factores ya clásico) creando nuevas centralidades económicas y nuevos corredores de flujo de personas y mercancías (Woessner, 2005). Estas áreas se

\footnotetext{
${ }^{6}$ Parece una ironía de la historia que fortalezas y castillos que en su día cerraban los territorios sean hoy atractivos turísticos de primer orden, destinados justamente a aumentar el tráfico de personas que un día debieron impedir. Véase en el apartado de referencias la red Les Fortaleses Catalanes, que estudia y promueve, también con carácter transfronterizo, estas edificaciones en la región de Cataluña. Por su parte, las edificaciones militares de Vauban figuran inscritas como Patrimonio de la Humanidad en la lista de la unESco (Les Fortaleses Catalanes, s.f).
} 
acaban configurando como "nuevas regiones" que se delimitan no por el gobierno sino por la economía y por lo tanto son verdaderas regiones funcionales en el sentido clásico de este término geográfico. Ello crea problemas no solo a la hora de definir unas entidades con base únicamente en lo económico (Ruffray et al., 2011) sino de legitimar estas nuevas divisorias vis a vis de las divisiones territoriales de la administración pública (o regiones formales). Al final, y según Dresser y Wilson (2006):

Transnationalism challenges traditional visions of the nation-state, notions of sovereignty, concepts of citizenship, forms of political participation, and definitions of class and community. Transnational communities are both a cause and a consequence of heightened integration, and their existence must be understood and taken into account by policy makers on both sides of the border (Dresser y Wilson, 2006, p. 1).

La gobernanza, que es el modus operandi por excelencia para pilotar estas regiones, emerge aquí no solamente como una buena praxis de acción pública o de concertación de actores, sino como una vía para legitimar a formas de territorialización que escapan a los modos clásicos de legitimación política. En la medida en que no dispone de estructuras de representación democrática, para legitimarse, una euroregión necesita poner en marcha mecanismos funcionales de gobernanza parecidos a los que inspiran la gobernanza multinivel de la ue (Morata y Noferini, 2011, p. 3). ${ }^{?}$

La dicotomía entre una frontera más cerrada y otra más abierta sigue, bajo distintas formas, en nuestros días. En efecto, la cuestión fronteriza puede verse desde el punto de vista de la seguridad o bien desde la integración económica. Incluso una misma frontera puede suscitar ambas miradas: por un lado Ginsburg (2010) o Dunn y Palafox (2005) y por el otro Vance (2012) o Brunet-Jailly (2006) leen, respectivamente, la frontera estadounidense bajo estas dos distintas perspectivas. Koff (2006) ofrece asimismo una excelente comparación entre ambas tendencias, en donde, según él, en EE. UU. predomina la visión de la seguridad mientras que en Europa, y en sus fronteras interiores, prevalece el tema de la integración económica. La cuestión de la mirada sobre la frontera y la representación que de esta tiene la sociedad que vive cercana a ella no es un tema trivial. Raffestin (1992) subraya la función de encaje social y nacional que tiene una frontera, y por lo tanto ve difícil el sueño europeo de una simple abolición de fronteras. En un escenario de fronteras cerradas o como zonas conflictivas, se subrayarán los problemas socioeconómicos (Díaz y Turner, 2012); las cuestiones de presión migratoria y su gestión (Ackerman y Furman, 2013; Campos, 2012; Chavel, 2013) o la pura delincuencia (Dube, Dube y García, 2013; García, Gaxiola y Guajardo, 2007), unas visiones que implican una idea militarista del espacio fronterizo, incluso a veces imposiciones en cuestiones de seguridad de la nación más fuerte a la más débil, lo cual no deja de irritar a algunos autores (Kent, 2011; Velasco, 2012).

Al final, la misma UE reconoce que la libre circulación de personas, bienes, servicios y capitales instaurada en el llamado espacio Schengen solo puede realizarse en paralelo a la iniciativa del espacio europeo de libertad, seguridad y justicia (ELSJ) (Fernández, 2004), demostrando así que la tensión entre libertad y seguridad está irresuelta en su fondo.

Un desarrollo interesante que tiene su origen en Europa en los años sesenta del siglo pasado es la cooperación transfronteriza. Dicha cooperación tiene ya su propia historia en algunas regiones, por ejemplo la de Basilea (c.f. la primera sección del presente artículo), en donde se alcanza el medio siglo (Kreis, 2012; Weber, Jakob y Basiliensis,

${ }^{7}$ Véase asimismo a Varela, Rojo y Sá Marques (2006) para una discusión sobre gobernanza en la zona transfronteriza hispano-portuguesa, denominada "Eixo Atlántico". 
2013). Por su parte, la corta historia de la apertura de fronteras en Europa, acaecida a principio de los años noventa tras el hundimiento del comunismo en Europa del Este, ha sufrido ya sus altos y bajos en este escaso cuarto de siglo de su existencia, lo que demuestra la pervivencia de la mencionada tensión: de unos primeros años de gran entusiasmo se ha pasado a un cierto escepticismo y a limitar progresivamente aspectos de la libre circulación de personas (Amoroso, 2013). Yndigegn (2011) con el provocador título "Between debordering and rebordering Europe", destaca la pendularidad de ideas respecto al tema a lo largo del último cuarto de siglo en la región escandinava de Oresund. Por su parte, Nikiforova (2010) tematiza la desaparición versus el refuerzo de fronteras que ha habido estos últimos años en la zona transfronteriza entre Polonia, Lituania y Bielorrusia. Y véase McGuire (2013) en donde se aborda el ciclo de rematerialización de la frontera Estados Unidos-México.

Cabe subrayar que en las últimas décadas en Europa, las esperanzas se han depositado en lo transfronterizo, sobre todo en momentos de un nuevo inicio histórico o social, como si la nueva apertura de regiones de frontera coadyuvara a este nuevo inicio y fuera la correspondiente territorial del ánimo social. Así, O'Dowd y McCall (2006) ven la cooperación transfronteriza como medio de refuerzo de la paz en Irlanda del Norte, y también Czyzewski ve en las prácticas transfronterizas una forma de recomponer la Europa Central, al respecto dice "I am inclined to agree with the opinion that working out a modern form of the borderland ethos will determine the future of Central Europe; it will define each integrative trend, including European integration" (Czyzewski, 2012, p. 173).

El estudio de la frontera y los territorios fronterizos puede abordarse desde muy distintas perspectivas científicas, reviste una complejidad no menor y tiene subáreas de estudio muy diferentes. Así, el concepto de frontera interior, relevante para muchos países latinoamericanos (Montero, 1997), se refiere a aquellos territorios que, estando dentro del país, muestran un bajo grado de colonización humana y por tanto de actividad económica, y constituyen unos territorios a someter. Borda (2011) hace una propuesta de organización territorial en una zona de estas características. Andrade (2004), por su parte, ilustra ampliamente los avatares de los territorios de frontera interior colombianos, afirmando en su trabajo que:

Colombia es uno de los países que todavía tienen abiertas grandes fronteras interiores de ocupación. Este hecho, sin embargo, representa una situación contradictoria. Si bien desde un punto de vista ambiental la persistencia a inicios del siglo xxi de grandes áreas silvestres o en las cuales predominan los ecosistemas naturales representa un activo de valor global, estas mismas áreas son el escenario de conflictos sociales no resueltos, y de los cuales depende en gran medida el futuro político del país (Andrade, 2004, p. 1).

Otra perspectiva científica de la frontera nos es dada por el concepto de frontera social, usado por autores ya hace años para definir espacios de exclusión económica colindantes con espacios más acomodados, muchas veces dentro de una misma urbe (Soulet, 2010). O bien existe el concepto de frontera mental, con el que, en tiempos recientes, estudiosos europeos intentan explicar cómo, a pesar de la política de fronteras abiertas en la UE, muchos ciudadanos deciden no explorar las ventajas que esta situación ofrece (Müller, 2014a y 2014b; Spierings y Velde, 2013). Estas últimas investigaciones se mueven ya en el ámbito de representaciones mentales colectivas (Clémence y Lorenzi-Cioldi, 2005).

Para finalizar la presente sección, distinguiremos brevemente los cuatro conceptos de territorialización de la cooperación entre países: llamaremos binacional a todo aquel trabajo, proyectos y acuerdos que dos países determinados (multinacional si son tres o 
más) llevan a cabo con base en la soberanía que poseen y a unos objetivos de interés común, pudiendo circunscribirse tales acciones a zonas geográficas determinadas, tengan lugar estas en los territorios fronterizos o no. Ejemplos de ello son acuerdos binacionales para explotación de recursos naturales. Así, encontramos que Castro, Cortez y Sánchez (2011) y el Acuerdo entre los Estados Unidos Mexicanos y los Estados Unidos de América relativo a los yacimientos transfronterizos de hidrocarburos en el Golfo de México de 2008 se examinan la explotación del agua y los hidrocarburos, respectivamente, en la región de frontera entre Estados Unidos y México. ${ }^{8}$ La existencia de tratados binacionales sobre temas de cooperación específicos en general, o sobre zonas de frontera en particular, no excluye la cooperación transfronteriza sino que puede tener lugar en paralelo a la misma.

Además de los tratados binacionales, algunos países tienen iniciativas determinadas en pro de sus regiones de frontera: el programa colombiano "Fronteras para la prosperidad", implantado por el gobierno del país hace pocos años y consistente en subvenciones a las regiones de frontera, es uno de los modos de paliar el "efecto frontera" dentro del propio país. No se trata de un programa transfronterizo ya que no se colabora con los países vecinos en su implementación, pero su mera existencia significa un reconocimiento del mencionado efecto en estas regiones colombianas por parte del gobierno (Ortiz, 2012, p.47 y ss.; Plan Fronteras para la Prosperidad, 2012). También en Venezuela existe la misma conciencia respecto a la marginalización de territorios fronterizos y la ayuda que precisan del Estado, lo cual incluso se ha inscrito en la constitución del país:

El Estado tiene la obligación de establecer una política integral en los espacios fronterizos, terrestres, insulares y marítimos, preservando la integridad territorial, la soberanía, la seguridad, la defensa, la identidad nacional, la diversidad y el ambiente, de acuerdo con el desarrollo cultural, económico, social y la integración. Atendiendo a la naturaleza propia de cada región fronteriza a través de asignaciones económicas especiales, una Ley orgánica de fronteras determinará las obligaciones y objetivos de esta responsabilidad (Constitución de la República Bolivariana de Venezuela, art. 15, 1999).

Sin embargo, Barrios y Rivas (2011) critican tanto el no-despliegue del citado mandato constitucional como la poca dinámica del gobierno de Venezuela hacia las regiones de frontera, lo cual ha causado un debilitamiento económico y de otros órdenes en tales regiones. Por su lado, Jiménez (2015) descubre interesantes y nuevas formas de gobernanza en la frontera colombo-venezolana, a pesar de la difícil situación actual entre ambos países.

A un nivel inferior del nacional, existe la cooperación interregional o transfronteriza, que es la que se da entre territorios de un país y los de otro, generalmente territorios colindantes del país vecino. Por otro lado, llamamos transfronterizo a las acciones y proyectos que tienen lugar en las áreas de frontera de dos o más países colindantes. La región aquí es claramente definida y nos viene dada por la proximidad a la frontera. Se puede introducir, si se quiere, el nivel transmunicipal que es la cooperación entre los municipios que están a caballo (o inmediatamente) de la zona fronteriza y en la que cabría englobar las áreas metropolitanas transfronterizas como las de Ginebra y Basilea mencionadas anteriormente. Estas diferentes escalas coexisten perfectamente las unas con las otras, y las únicas cuestiones que plantean es la elección óptima de ámbitos territoriales e instrumentos de gobernanza a usar para cada proyecto.

\footnotetext{
${ }^{8}$ Estos y otros ámbitos pueden ser abordados también desde un enfoque transfronterizo y no sólo desde el binacional. Así, Uitto y Duda (2002) proponen tal enfoque para resolver el tema de los acuíferos multinacionales.
} 
Sin embargo, además de la colaboración a escala interregional o transfronteriza, la gran novedad aportada por la cooperación territorial en la UE es la cooperación en red, es decir, la cooperación que se establece entre aquellos territorios que no tienen contigüidad geográfica pero sí fortalezas o intereses comunes. Nos encontramos entonces ante una innovativa forma de cooperación territorial. Es la que une por ejemplo a las ciudades europeas de la cerámica o bien la que conecta varias islas mediterráneas en el proyecto Archimed. En el primer caso se trata de una cooperación muy concreta en un sector económico, en el otro caso se trata de una cooperación también política y de lobby, ya que consiste en la intención conjunta de las islas mediterráneas de la uE en hacer oír su voz ante esta.

En resumen, el advenimiento de la cooperación transfronteriza ha coadyuvado a cambiar enormemente el paradigma de frontera existente hasta mediados del siglo pasado - empujando de modo decisivo en Europa primero y en otras zonas del mundo más tarde- de lo regional (entendido como región formal) a lo territorial, según lo apunta Ramírez (2011). Listamos en la Tabla 1 estos cambios, indicando las evoluciones sufridas a distintos niveles.

Tabla 1: Cambios en el paradigma de frontera de lo regional a lo territorial

\begin{tabular}{|l|c|l|}
\hline Frontera como punto o línea & $\rightarrow$ & Frontera como espacio o región \\
\hline Zona de disrupción, cerrada & $\rightarrow$ & Zona de paso, abierta \\
\hline Prioridad a la seguridad & $\rightarrow$ & Prioridad al desarrollo económico \\
\hline Autoridad de gobierno & $\rightarrow$ & Autoridad de gobernanza \\
\hline Gestión piramidal y contigua & $\rightarrow$ & Gestión multiescalar y en red \\
\hline Territorialización formal-administrativa & $\rightarrow$ & Territorialización social-económica \\
\hline Cultura central, mainstream & $\rightarrow$ & Cultura periférica, bordering \\
\hline Representación del espacio nacional & $\rightarrow$ & Representación de espacios continuos \\
\hline Estereotipos de los pueblos vecinos & $\rightarrow$ & Ausencia de estereotipos de los vecinos \\
\hline $\begin{array}{l}\text { Objeto de estudio preferente del derecho, la po- } \\
\text { lítica o la defensa }\end{array}$ & $\rightarrow$ & $\begin{array}{c}\text { Objeto de estudio preferente de la geografía, } \\
\text { la economía o la sociología }\end{array}$ \\
\hline
\end{tabular}

Fuente: Elaboración propia.

\section{Territorialidad y cotidianidad en las zonas fronterizas de Europa}

La presente sección resume algunos de los debates actuales sobre la integración de las zonas fronterizas de Europa, relacionados con el ámbito de la territorialidad y los comportamientos espaciales de los habitantes de las regiones de frontera, unos debates que se hallan abiertos y por tanto serán objeto de reflexiones e investigaciones en el futuro.

Hay tres áreas en que los habitantes de las zonas fronterizas aprovechan de modo especial las ventajas económicas que, en su espacio, ofrece la apertura de fronteras y la integración económica como la que se ha dado en la UE en el último medio siglo: el trabajo, la vivienda y las compras. En el primer caso, siempre veremos flujos de trabajadores transfronterizos hacia el país que paga unos salarios más elevados. Ejemplo de ello es el espacio transfronterizo entre Francia, Alemania y Suiza: el primer país es emisor neto de trabajadores mientras que el último es receptor neto, a pesar de que en teoría el intercambio puede ser bidireccional. Los otros dos casos -vivienda y compras- suscitan el 
movimiento hacia el país que ofrece los precios más baratos. Ejemplo del primer caso son los alemanes de la zona fronteriza con Austria adquiriendo combustible para uso particular en ese país. Ejemplo del caso de la vivienda es la frontera entre Bélgica y Alemania, con numerosos alemanes que residen en el lado belga, aun teniendo sus centros de vida del lado alemán de la frontera, y ello por los precios inmobiliarios más bajos. Estas situaciones se han replicado en todas las fronteras europeas en momentos de apertura, siempre que el diferencial económico de países colindantes sea lo suficientemente alto como para motivar los movimientos transfronterizos que se mencionan.

Evidentemente, la integración regional por las fronteras no depende sólo de la apertura de las mismas como es el caso en el espacio Schengen sino que factores lingüístico-culturales son determinantes: si se habla la misma lengua al otro lado de la frontera, la integración regional transfronteriza es más fácil. La orografía tiene también su peso, haciendo algunas fronteras más impermeables que otras, sin embargo este factor es secundario respecto del lingüístico y cultural.

A continuación se proponen tres temas para discusión y que forman parte del debate científico actual sobre integración transfronteriza en Europa:

1. La integración en zonas transfronterizas debe ser más social que económica: de acuerdo con Müller (2014b), quien tiene razón cuando dice que el factor económico, por sí solo, no es capaz de unir por encima de las fronteras. A pesar de que lo económico puede iniciar procesos transfronterizos que a corto o a medio plazo tengan una masa crítica de personas concernidas (básicamente trabajadores o compradores), no es hasta que lo social entra en escena (ocio compartido, clubs deportivos compitiendo, escuelas haciendo intercambios, asociaciones culturales compartiendo programas, etcétera) que el transfronterizo se vuelve real y duradero. En efecto, los precios bajos o los productos que se puedan adquirir al otro lado están sujetos inevitablemente a una volatilidad: lo que hoy es más barato a un lado de la frontera puede serlo mañana en el otro. Y cierto es que la relación comprador-proveedor no es de lo más duradero, mientras que las relaciones sociales sí lo son, independientemente de fluctuaciones económicas. Por tanto, se trata de crear espacios de intercambio humano y comunión social en las zonas transfronterizas.

2. Es preferible que los procesos transfronterizos sean bottom-up y no top-down: no pocas veces en la UE hemos visto grandes concertaciones de administraciones o gobiernos de diferente nivel, que sin embargo han dado lugar a proyectos faltos de masa crítica. Es decir, se han puesto recursos donde al final se han beneficiado pocos ciudadanos ya que el motor de los proyectos procedía de arriba y no de abajo. Ciertamente, es opinión de este autor que son necesarias personas entusiastas de la causa transfronteriza en el seno de la administración pública, para hacer avanzar la integración. Sin embargo, el entusiasmo de unos pocos, aunque tengan autoridad, no basta: es necesario concebir proyectos que puedan tocar potencialmente a muchas personas, que de este modo darán al nivel administrativo europeo una visibilidad mucho mayor que ahora. El proyecto transfronterizo alrededor de la ciudad de Ginebra, uno de los más antiguos de la UE (a pesar de que la misma Suiza no pertenece a la UE), ha conseguido un eco notable justamente por esto: las oportunidades laborales de Ginebra atrajeron a trabajadores franceses, ya desde los años sesenta, quienes, aun trabajando en Suiza, preferían vivir en su país y por tanto esto fue el inicio de una exitosa historia transfronteriza. Las autoridades tuvieron un papel no de iniciador sino de acompañamiento y regulación a posteriori: así hay que entender el acuerdo 
entre Suiza y Francia en los años setenta por el que el cantón de Ginebra transfería parte de los impuestos recaudados a sus trabajadores transfronterizos franceses a los municipios en donde estos residían, a fin de que dichos municipios pudieran construir infraestructuras acordes a una población en aumento (Saint-Ouen, 2012).

3. Nuestros hábitos geoespaciales nos determinan fuertemente: otro modo de decir que "somos animales de costumbres" y estas son las que son. Estamos marcados por unos hábitos contraídos a lo largo de nuestra vida y también por los hábitos geoespaciales de nuestro entorno. Es normal, pues, que si algo se ha hecho de un modo "toda la vida" se siga haciendo así hasta que haya un cambio grande de los paradigmas válidos. Todo ello para decir que un hábito de generaciones y siglos no se rompe en unos lustros. La cotidianidad en los hábitos juega un rol fundamental aquí, pero por ambos lados de la frontera. Así, si alguien se crea el hábito de traspasar una frontera por asuntos de ocio, es probable que así lo siga haciendo hasta que no haya un cambio lo suficientemente importante para justificar una revisión de este hábito. Por lo demás, adentrarse en el terreno desconocido, más allá de la frontera, en un país que quizás hace un siglo era enemigo del propio, y que tiene ya sea lengua, reglas o costumbres diferentes (y a veces las tres cosas), es algo que, socialmente, se hace poco a poco: primero gracias a quien tiene la curiosidad y la visión de ser un explorador y después con los grupos sociales que siguen a estos exploradores hasta -si es el casollegar a una masa crítica. Estos procesos requieren tiempo y energías y sólo se pueden favorecer, y no pilotar, desde arriba. De hecho, en esta dirección van los estudios que buscan ahora una mayor masa de personas que usen las ventajas transfronterizas que propone la UE, y que explican la relativamente baja cifra de personas concernidas por el transfronterizo: véase Müller (2014a y 2014b) o los trabajos de investigación de la Universidad de Nimega, Países Bajos (Spierings y Velde, 2013) resumidas en la palabra-clave unfamiliarity. ${ }^{9}$

\section{Conclusión}

La presente contribución ha pretendido dar una aproximación histórico-social y funcional al tema de las fronteras a partir de una primera reflexión que es la crisis en la que, en los últimos años, parece estar sumida la política europea de fronteras. Ello se constata a partir de la actual situación de desavenencias entre países gestionando la llegada de refugiados de Medio Oriente, o bien las dificultades que presenta Suiza en su integración al espacio Schengen. Tras una revisión de la literatura, se han expuesto brevemente los temas de debate actuales sobre las fronteras en la Unión Europea, así como las prácticas transfronterizas de sus habitantes. Hacia estas últimas es donde la academia dirigirá sus estudios en los próximos tiempos. Como conclusión, se puede afirmar que ha habido una tendencia —no siempre lineal- hacia un suavizamiento de las fronteras y una mayor integración de las zonas fronterizas de la UE, pero que actualmente se está en una serie

\footnotetext{
${ }^{9}$ Existe multitud de propuestas hechas desde diferentes ámbitos económicos, sociales, políticos o culturales para la mejora de la efectividad de la cooperación transfronteriza y multiplicar sus resultados. Destacan las propuestas de la Conference of European Cross-border and Interregional City Networks [CECICN] (2012) sobre la mejora de la cooperación para convertirla en cooperación territorial de segunda generación o cooperación territorial smart.
} 
de disyuntivas - presentadas a lo largo del artículo- que cuestionan marcos generales aplicados hasta el momento y que apuntan a un aumento de la discusión en torno a este ámbito para los próximos años.

\section{Referencias}

Abellán, L. (9 de febrero de 2014). Suiza da un portazo a la Unión Europea. El País. Revisado de http://internacional.elpais.com/internacional/2014/02/09/actualidad/1391942183_207520.html

Ackerman, A. R. y Furman, R. (2013). The criminalization of immigration and the privatization of the immigration detention: implications for justice. Contemporary Justice Review: Issues in Criminal, Social, and Restorative Justice, 16(2), 251-263.

Amoroso, M. (29 de julio de 2013). Contrôles aux frontières nationales: les pays membres de l'espace Schengen ont trouvé jeudi un compromis avec le PE et la Commission [Mensaje en un blog]. Recuperado de http://europe-liberte-securite-justice. org/2013/07/29/controles-aux-frontieres-nationales-les-pays-membres-de-lespaceschengen-ont-trouve-jeudi-un-compromis-avec-le-pe-et-la-commission/

Andrade, G. (2004). Selvas sin ley. Conflicto, drogas, globalización de la deforestación de Colombia. En Colombia. Guerra sociedad y medio ambiente (pp.107-174). Colombia: Foro Nacional Ambiental, Fundación Friedrich Ebert Stiftung de Colombia.

Aussilloux, V. (2016). Les conséquences économiques d'un abandon des accords de Schengen. La note d'anayse, (39). Recuperado de http://www.strategie.gouv.fr/sites/strategie. gouv.fr/files/atoms/files/note_danalyse_ndeg39.pdf

Barrios, N. y Rivas, N. (2011). La frontera Táchira-Norte de Santander: Escarpada a la integración entre acuerdos y crisis. Revista Instituto Altos Estudios Europeos, (0), 22-35.

Bassets, L. (27 de marzo de 2014). Celos soberanos. El País. Recuperado de http://internacional.elpais.com/internacional/2014/02/12/actualidad/1392229738_505048.html

Bassols, N. (2010). Un àmbit de creixement econòmic: L'Espai Català Transfronterer. Generalitat de Catalunya. Treballar a la Frontera. La Generalitat a Perpinyà $i$ a l'Alguer (pp. 46-55). Recuperado de http://www.slideshare.net/maritzeta/treballar-a-la-frontera

Bertinat, J., Calvette, A., Ignatczuk, M. I., Sauval, M. y Villamil, L. (2012). Acercamiento al sistema centro-periferia, Cátedra de crecimiento y desarrollo económico. Recuperado de http://www.ccee.edu.uy/ensenian/catcrecydes/2012-10-30_Acercamiento\%20 al\%20sistema\%20centro-periferia.pdf

Borda, S. (2011). Ordenamiento territorial en una frontera de colonización: entre la legitimación normativa y la legitimación social. Análisis de caso: los colonos del interfluvio Losada-Guayabero (Tesis de Maestría). Universidad del Tolima, Ibagué, Colombia.

Bottino, M. R. (2009). Sobre límites y fronteras. Estudios Históricos, (1). Recuperado de http:// www.estudioshistoricos.org/edicion_1/maria-bottino.pdf

Brunet-Jailly, E. (2006). NAFTA and cross-border relations in Niagara, Detroit and Vancouver. Journal of Borderlands Studies, 21(2), 1-20.

Bustamante, A. M. y Chacón G., E. J. (2013). Formas identitarias en la región fronteriza de Táchira (Venezuela)-Norte de Santander (Colombia). Desafíos, 25(1), 165-203.

Campos, A. (2012). Vis a vis: Tijuana, México y Tecún Umán, Guatemala. Jóvenes, movilidad e imaginarios de frontera. Identidades, 3(5), 84-99. 
Castro, J. L., Cortez, A. A. y Sánchez, V. (2011). Gestión del agua en cuencas transfronterizas México-Estados Unidos: algunos elementos conceptuales para su estudio. Aqua-LAC, 3(2), 105-114.

Chavel, S. (2013). Le migrant et la frontière. Terrains de l'affrontement moral. En E. Ferrarese (Ed.), Qu'est-ce que lutter pour la reconnaissance? (pp. 187-208). Lormont, Francia: Éditions Le Bord de l'eau.

Clémence, A. y Lorenzi-Cioldi, F. (2005). Dinámica de la representación. De las representaciones mentales a las representaciones sociales de los grupos. Trayectorias, VII(18), 64-78.

Comte, H. y Levrant, N. (2006). Aux coutures de l'Europe: Défis et enjeux juridiques de la coopération transfrontalière. París, Francia: L'Harmattan.

Conference of European Cross-border and Interregional City Networks (CECICN). (Junio de 2012). Cities and regions cooperating across borders: an opportunity to overcome crisis. En Conclusions from the 1st Congress on Smart Cooperation, La Coruña, España.

Czyzewski, K. (2012). Reinventing Central Europe. En L. Donskis (Ed.), Yet another Europe after 1984. Rethinking Milan Kundera and the idea of Central Europe (pp. 171-182). Amsterdam, Países Bajos: Rodopi.

Díaz, E. y Turner, E. (2012). Pobreza y política social en México y estados de la frontera norte. Análisis Económico, ххиII(64), 23-46.

Dresser, D. y Wilson, V. (2006). U.S.-Mexico relations: Permeable borders, transnational communities. Los Ángeles, Estados Unidos de América: Pacific Council for International Policy,

Dube, A., Dube, O. y García, O. (2013). Cross-border spillover: u.s. gun laws and violence in Mexico. American Political Science Review, 107(3), 397-417.

Dubois, Y. y Rérat, P. (2012). Vivre la frontière: les pratiques spatiales transfrontalières dans l'Arc jurassien franco-suisse. Belgeo, 1-2, 1-14.

Dunn, T. J. y Palafox, J. (2005). Militarization of the border. En S. Oboler y D.J. González. The Oxford Encyclopedia of Latinos and Latinas in the United States. Oxford University Press. doi: 10.1093/acref/9780195156003.001.0001

Fawcett, C. W. (1918). Frontiers: a study in political geography. Oxford, Gran Bretaña: Clarendon Press.

Fernández, J. C. (2004). El espacio de libertad, seguridad y justicia consolidado por la Constitución Europea. Revista Jurídica Española La Ley, (4), 1867-1881.

García, N. J., Gaxiola, E. G. y Guajardo, A. (2007). Movimientos transfronterizos México-Estados Unidos: Los polleros como agentes de movilidad. Confines, 3(5), 101-113.

Gibler, D. M. (2012). The territorial peace. Cambridge, Gran Bretaña: Cambridge University Press.

Ginsburg, S. (2010). Securing human mobility at the U.s.-Canada border. National Strategy Forum Review, 9(3), 1-7.

Harguindéguy, J.-B. (2007). La frontière en Europe: un territoire? Coopération transfrontalière francoespagnole. París: L'Harmattan.

Hinfray, N. (2010). Les espaces transfrontaliers, nouveaux territoires de projet, nouveaux pöles de développement au sien d'une Europe polycentrique? (Tesis doctoral). Université François Rabelais, Tours, Francia. 
Jiménez, C. M. (2015). Gobierno local en la frontera colombo-venezolana en medio del distanciamiento binacional. Análisis Político, 28(83), 55-72.

Kent, J. (2011). Border bargains and the "new" sovereignty: Canada-us border policies from 2001 to 2005 in perspective. Geopolitics, 16(4), 793-818.

Koff, H. (2006). On the cutting edge: Border integration and security in Europe and North America (EUI Documentos de trabajo, Nro. 21). Florencia, Italia: European University Institute.

Koff, H. (2008). Las políticas fronterizas comparadas y las estructuras del poder. Estudios Políticos, 32, 119-134.

Kohli, A. (10 de febrero de 2014). Zurück in die neunziger Jahre. Neue Zürcher Zeitung. Recuperado de http://www.nzz.ch/aktuell/schweiz/zurueck-in-die-neunziger-jahre-1.18240098

Kreis, G. (2012). Grenzüberschreitende mikrointegration. Der basler dreiländerraum gestern-heute-morgen. En Autor, Baslerschriften zur europäischen integration, (Nro. 100). Basilea, Suiza: Europainstitut der Universität Basel.

Les Fortaleses Catalanes. (s.f.). Presentación. Recuperado de https://lesfortalesescatalanes. info/la-fundacion/presentacion/

Márquez, R. y Romo, H. D. (Eds.) (2008). Transformations of la familia on the U.S.-Mexico Border. Notre Dame, Indiana: University of Notre Dame Press.

McGuire, R. H. (2013). Steel walls and picket fences: Rematerializing the U.s.-Mexican border in ambos Nogales. American Anthropologist, 115(3), 466-480.

Moncusí, A. (2008). Fronteres i evolucions locals. El cas de la Cerdanya. Mirman$d a$, (3), 58-67. Recuperado de http://raco.cat/index.php/Mirmanda/article/ view/250585/335357

Montero, P. (1997). Chile: nuevo concepto de fronteras interiores. Colombia: Sociedad Geográfica de Colombia, Academia de Ciencias Geográficas.

Morata, F. y Noferini, A. (2011). Gobernanza y capacidades institucionales en la frontera pirenaica. Trabajo presentado en el xi Congreso Español de Ciencia Política y de la Administración, Barcelona, España.

Müller, N. (2014a). Die alltägliche reproduktion nationaler Grenzen. Konstanz, Alemania: Uvк.

Müller, N. (2014b). Europ. Gespräche: Die alltägliche reproduktion nationaler Grenzen [Archivo de audio]. Entrevista en el programa Kontext de la radio suiza SFR2. Recuperado de http://www.srf.ch/sendungen/kontext/europ-gespraeche-die-alltaegliche-reproduktion-nationaler-grenzen

Nikiforova, B. (2010). Strengthening and disappearing borders in the Lithuanian-Polish-Belarusian borderland. LIMES Cultural Regionalistics, 3(1), 4-5.

O'Dowd, L. y McCall, C. (2006). The significance of the cross-border dimension for promoting peace and cooperation (Documentos de trabajo en British-Irish Studies núm. 55). Irlanda: Institute for British-Irish Studies, University College Dublin.

Ortiz, D. M. (2012). El rol de las fronteras en la integración regional: caso frontera andina colombo-ecuatoriana (Tesis de maestría). Universidad Andina Simón Bolívar, Sede de Ecuador.

Petrakos, G. y Topaloglou, L. (2006). Economic geography and European integration: The effects on the Eu external border regions. Discussion Papers Series, 12(8), 153-174.

Poch, R. (2014). Grieta en el Eurodistrito del Rin [Mensaje en un blog]. Recuperado de http:// blogs.lavanguardia.com/berlin-poch/grietas-en-el-eurodistrito-del-rin-83462

Raffestin, C. (1992). Autour de la fonction sociale de la frontière. Espaces et sociétés, (70/71), 157-164. 
Ramírez, B. R. (2011). Space and politics in territorial development. Economía Sociedad y Territorio, 11(37), 553-573.

Regio Basiliensis. (2015). Drei Länder zwei Sprachen ein Lebensraum: grenzüberschreitende Modellregion Oberrhein. Recuperado de http://www.regbas.ch/de/assets/ File/downloads/Basis_PPP_GueZ_2015.pdf

Romo, R. (1987). Boder Culture. En The World and I (pp. 479-496).

Ruffray, S. de, Hamez, G., Grasland, C., Lambert, N., Hamm, A. y Gallet-Moron, E. (2011). Enjeux des territoires frontaliers à l'échelle nationale. Vers la détermination et la délimitation de pôles transfrontaliers. Francia: ums RIATE, DATAR.

Sahlins, P. (1990). Natural frontiers revisited: France's boundaries since the seventeenth century. The American Historical Review, 95(5), 1423-1451.

Saint-Ouen, F. (2012). Cross-border cooperation between Geneva and neighbouring France: From sectorial to global. Suiza: Institut Européen, Université de Genève.

Schuler, M. (2006). Territoires et réseaux. Frontières et limites. Suiza: Institución INTER-Chorôs.

Soulet, M. H. (2010). Frontières sociales, frontières culturelles, frontières techniques. SociologieS. Recuperado de http://sociologies.revues.org/3304

Spierings, B.H.A. y Velde, B.M.R. van der (2013). Cross-border mobility, unfamiliarity and development policy in Europe. European Planning Studies, 21(1), 1-4.

Uitto, J. I y Duda, A. H. (2002). Management of transboundary water ressources: Lessons from international cooperation for conflict prevention. The Geographical Journal, 168(4), 365-378.

uRbaCt (2010). Conference finale du project URBACT "EGCT", Reporte final. Recuperado de http://urbact.eu/sites/default/files/import/Projects/Egtc/documents_media/ CR_Conf_fin_Esztergom_Mai_2010_EGTC_URBACT_FR.pdf

Vance, A. (2012). Crossing bridges: Observations and strategies by cross-border business communities in an evolving regulatory environment (Documentos de trabajo Nro. 18).Washington, Estados Unidos de América: Border Policy Research Institute, Western Washington University.

Varela, E. J., Rojo, A., Río, J. A. y Sá Marques, T. (2006). A governança na Euro-regiao Galicia-Norte de Portugal. Vigo, España: Eixo Atlântico do Noreste Peninsular.

Velasco, J. C. (2012). Movilidad humana y fronteras abiertas. Claves de Razón Práctica, (219), 28-35.

Weber, M., Jakob, E. y Regio Basiliensis. (Eds.) (2013). Die regio-idee: Grenzüberschreitende zusammenarbeit in der region basel. Basilea, Suiza: Christoph Merian Verlag.

Wikipedia. (2014). Swiss immigration referendum, February 2014, Results by canton [mapa]. Recuperado de https://en.wikipedia.org/wiki/Swiss_immigration_referendum,_February_2014\#/media/File:Anti-Einwanderungsinitiative_2014.svg

Williams, J. (2006). The ethics of territorial borders. Nueva York, Estados Unidos de América: Palgrave Macmillian.

Woessner, R. (2005). Les villes et les régions françaises au défi de l'ouverture des frontières. Francia: IUFM d'Alsace, CRESAT-UHA.

Yndigegn, C. (2011). Between debordering and rebordering Europe: Cross-border cooperation in the Øresund region or the Danish-Swedish border region. Eurasia Border Review, 2(1), 47-59. 


\section{Material legislativo}

Acuerdo entre los Estados Unidos Mexicanos y los Estados Unidos de América relativo a los yacimientos transfronterizos de hidrocarburos en el Golfo de México [Secretaría de Relaciones Exteriores]. Suscrito el 20 de febrero de 2008 y ratificado el 12 de abril de 2008.

Constitución de la República Bolivariana de Venezuela, art 15, 1999.

Plan Fronteras para la Prosperidad. Resumen ejecutivo proyecto de inversión. Vigencia 2012. [Ministerio de Relaciones Exteriores]. 2012.

\section{Agradecimientos}

Agradezco a Jahir Lombana (UniNorte Barranquilla) y a Wilson Ladino (ESAP Bogotá) la información y comentarios dados sobre los conceptos geoeconómicos de "triángulo de oro" y "polígono de oro".

Narcís Bassols

Español. Ha sido técnico profesional en el proyecto Pyrémed-Pirimed de la Unión Europea. Doctorando de la Universitat Rovira i Virgili, Tarragona, España; reside en Barranquilla, Colombia y forma parte del Programa de Administración de Empresas Turísticas y Hoteleras, Facultad de Ciencias Económicas, Administrativas y Contables, Universidad Autónoma del Caribe. Líneas de investigación: turismo en zonas de frontera, el turismo urbano y cultural así como la aplicación a estas áreas de las nuevas tecnologías de la información y la comunicación. Publicación reciente: "Branding and promoting a country amidst a long-term conflict. The case of Colombia" en Journal of Destination Marketing and Management, 2016.

José Rafael Correa Fernández

Colombiano. Maestría en Dirección de Organizaciones en la Economía del Conocimiento, Universidad Oberta de Cataluña, Barcelona, España. Actualmente es profesor de tiempo completo en la Escuela de Administración, Corporación Unificada Nacional de Educación Superior, Seccional Santa Marta, Colombia. Líneas de investigación: la internacionalización de ONG's y el turismo en zonas de frontera. Publicación reciente: Marketing y comunicación de onGs: el caso de Caritas Diocesana de Girona (Tesis de maestría), Universidad Oberta de Cataluña, Barcelona, 2014. 